\title{
Pathomorphosis of Uterine Myomas in Women
}

\author{
Paweł Madej ${ }^{*}, 1$ and Janusz A. Madej ${ }^{2}$ \\ ${ }^{I}$ Department of Gynecological Endocrinology, Silesian Medical University, ul.Medyków 14, 40-752 Katowice, Poland \\ ${ }^{2}$ Department of Pathological Anatomy, Pathophysiology, Microbiology and Forensic Veterinary Medicine, Wroctaw \\ University of Environmental and Life Science, ul.Norwida 31, 50-375 Wroctaw, Poland
}

\begin{abstract}
Taking stroma (ECM-extracellular matrix) and not tumour parenchyma as a criterion of categorization, the tumours can be divided into two groups, i.e. those which produce stroma (non-epithelial malignant tumours) or sarcomas and the tumours which take advantage of the local tissue stroma (malignant epithelial tumours) or carcinomas. Involvement of stroma is noted also within the reciprocal relationship between stroma and tumour cells, which has been described in detail on the example of uterine myoma. ECM also "collaborates" with CAM (cell adhesion molecules), particularly in development of neoplastic metastases.

Pathomorphosis of myomas, myosarcomas and of "stromal" uterine tumours was described with particular attention given to differential diagnosis and the resulting clinic predictive and prognostic implications. A probable mechanism of neoplasia based on dissipative structures of cells was presented and introduction of a disoric zone of a tumour, i.e. of a marginal zone between the tumour and the morphologically normal tissue was suggested. The zone seems extremely important in prediction and prognosis related to relapse and/or tumour metastases.
\end{abstract}

Keywords: ECM-extracellular matrix, CAM -cell adhesion molecules, myomas, myosarcomas, mechanism of neoplasia, dissipative structures, disoric zone, clinic predictive.

Every tumor consists of parenchyma and stroma, with few exceptions (hibernoma, chorioepithelioma) when the tumour is devoid of stroma. Classification of the tumour is determined by cells of parenchyma but condition of stroma, and vascular supply, infiltration with lymphocytes or its absence in particular affect the fate of parenchymal cells [1]. Thus, the connective tissue interstitium forms an integral component of tumour texture and, in parallel, represents a portion of body tissues in which the tumour develops. The stroma binds and nourishes the tumour and forms an inseparable complex with tumour epithelium, i.e. modelling tumour growth and differentiation. Apart from the cells, the stroma forms the other component of tissues and it consists of intercellular substance or extracellular matrix (ECM), which may involve a liquid (blood, lymph), gel and/or moulded elements, i.e. fibres. Numerous blood vessels are also here present [2].

Stroma is formed from connective tissue, originating from mesenchyme and this was formed from mesoderm, consists of the ground substance, fibres and cells (fibroblasts, mast cells, plasmacytes and migratory cells or lympho- and granulocytes). ECM is produced by fibroblasts or their analogues, e.g. osteoblasts, and less frequently by smooth myoblasts [3].

In epithelial tumours of carcinoma type stroma is formed by the local connective tissue, colonized by the tumour and working as "a slave" supplying needs of its owner. In turn sarcomas or mesenchymal tumours produce their own

*Address correspondence to this author at the Department of Gynecological Endocrinology, Silesian Medical University, ul.Medyków 14, 40-752 Katowice, Poland; Tel/Fax: (48) 32-252-82-21; E-mail: pmadej@interia.pl and pmadej@slam.katowice.pl stroma by themselves and need for the purpose no foreign, local tissue. This is reflected by the fact that carcinomas almost always are accompanied by an inflammatory infiltrate, consistent with the histogenetic incompatibility between the epithelial tissue and the locally colonized mesenchymal tissue, which is not noted in cases of sarcomas. In the latter case, the two types of connective tissue exhibit with each other a more pronounced histogenetic compatibility. Therefore, taking stroma and not the usually applied for the purpose parenchymal cells as the criterion for classification, two groups of tumours should be distinguished, including:

1- Tumours which produce stroma (non-epithelial malignant tumours) or sarcomas and

2- Tumours which take advantage of local tissue stroma (epithelial malignant tumours), or carcinomas [2].

Tumour stroma may involve a proper stroma or stroma induced by the tumour or it may consist of tissue in which the tumour grows. In a non-malignant tumour a harmony exists between parenchyma and stroma but in malignant tumours the process may be disturbed. Rich stroma as a rule accompanies epithelial tumours while in mesenchymal tumours the stroma is scanty and frequently restricted to the capillary network only. Occasionally, epithelial tissue or tumour parenchyma stimulates stroma to proliferation and the desmoplastic carcinoma develops (carcinoma desmoplasticum scirrhosum s. durum s. scirrhus). The parallel proliferation of stroma and epithelium, e.g. in adenoma, leads to development of fibroadenoma. Occasionally, bone tissue is formed in tumour stroma (ca osteoplasticum) or mucus acumulates in the stroma (ca gelatinosum), the mucus which originates from cells destroyed due to excessive secretion of the mucus. Also the stroma may undergo hyaline, calciferous or mucoid degeneration while the blood vessels may form 
dilatations (tumor teleangiectaticum s. cavernosum). Destruction of parenchyma may be accompanied by development within the stroma of small cysts (tumor cysticum), or melanin accumulates between the cells. When accompanied by oedema the stroma may become loose, contains blood vessel-resembling fissures lined with endothelial cells and individual capillary vessels due to which the entire pattern mimics that of foetal type tissue [1]. A loss of fibroplastic properties, in turn, e.g. in fibrosarcoma leads to development of a tumour which completely or almost completely is devoid of fibres of connective tissue. It should also be added that in metastatic foci both parenchyma and stroma develop but the parenchyma develops from neoplastic cells of the primary focus while stroma is formed by the stroma of an organ in which the metastasis develops.

Finally, it should be recalled that the young stroma with a good blood supply facilitates access of ionizing radiation to the neoplastic tissue, in contrast to hyaline, calciferous stroma or stroma consisting of chondrous or osseous tissue. Irradiation results in stromal alteration, e.g. to hyalinisation or cicatrization with atrophy of blood vessels, which explains failures of radiotherapy in relapses of tumours which were earlier treated with radiotherapy.

In neoplastic stroma reactive processes are being encountered, including fibroblast proliferation, hyalinization of collagen fibres, accumulation of infiltrates of lymphocytes and plasmacytes [1]. Presence of cell infiltrate in the stroma, in carcinomas in particular, reflect protective tendencies of the host and used to accompany fibroplasia. It leads to partial destruction of neoplastic cells (the so called self-control), but it may facilitate migration of the cells, inducing metastases. Neoplastic antigens may induce inflammatory reaction resulting in tissue oedema and destruction, which facilitates penetration of neoplastic cells to tissue spaces opened up by the inflammatory process. In such a situation, incomplete turnover of metabolic compounds takes place in ECM with accumulation of lactic acid and fatty acids, amino acids, accompanied by a decrease in $\mathrm{pH}$ to around 6 . Cumulation of the compounds lead also to increase in osmotic pressure while infringement of cell membrane integrity leads to release of enzymes out of the cells, e.g. of lysosomal enzymes, histamine and of prostaglandins which "leak out" to ECM [4].

ECM "collaborates" with cell adhesion molecules (CAM), including selectins, cadherins, integrins, IgCAM and other not only in physiological processes but also in pathology, e.g. in development of tumours and, in particular, in development of metastases [3]. The process of development of metastases of neoplastic cells represents a very complex phenomenon. Lack of cadherin E or catenin expression induces atrophy of occluding junctions and the cell becomes "free" which facilitates its migration to a blood vessel and, then, to penetrate other tissues. On its way the cells transgresses ECM, basement membrane of the blood vessel and endothelial cells. The aim is facilitated by metalloproteinases (MMP), which decompose collagen and other proteins. In turn, integrins of a neoplastic cell allow it to attach to laminin of the vascular basement membrane while collagenase IV destroys the membrane [5]. Membrane glycoproteins of a neoplastic cell, with, e.g. sialyl - Lewis - x groups, bind to selectins $\mathrm{E}$ of endothelial cells, which activates in- tegrins of the tumour cell forming a stronger junction with IgCAM of endothelial cells. This promotes migration of the neoplastic cells into and beyond the vascular wall [3].

\section{ECM IN UTERINE MYOMAS}

Tumours particularly rich in ECM include uterine myomas in women [6]. This is expressed by the increased content in the ECM of collagen [7], fibronectin, proteoglycanes and glycosaminoglycanes (GAG), which results in an increased weight of the tumour [8]. At the same time it should be stressed that smooth myocytes can synthesize collagen and proteoglycan proteins due to their rough sarcoplasmic reticulum. ECM affects also proliferation of smooth myocytes and their differentiation and represents the site of cumulation of growth factors and cytokines. Fujita [9] notes that uterine myomas contain by $50 \%$ more ECM than normal myometrium does and, therefore, they are occasionally termed fibroids. This overexpression of ECM and of collagen in particular is encountered only in the follicular phase of menstrual cycle [10]. It is accompanied by increased amounts of GAG and of proteoglycans in myomas, as compared to normal myometrium.

In myomas the overexpression includes mRNAs for collagens type I and type III, and the collagen itself changes not only its structure but also spatial orientation, as compared to normal myometrium, independently of tumour size and phase of menstrual cycle [11]. The changes collagen structure is induced probably by proteoglycans. The diameter of collagen fibres does not differ from that in normal muscles. Transfection of myoma myocytes in vitro using p53 inhibited production of collagen type I while no such changes were observed following transfection with p21 [4]. No differences were noted in amounts of total collagen and GAG between myomas from homozygotically negative females and heterozygotic females with Ehlros-Danlos type IV syndrome (EDS-IV), i.e. autosomal dominating disturbance, manifested by decreased amounts of collagen type III in ECM [4]. Myomas in women suffering from the syndrome manifest lowered content of collagen type III, consistent with the phenotype of the disease.

Cumulation of ECM results not only from its excessive production as related to its decomposition but also it's less extensive degradation by MMP. Activity of the enzymes remains under control of their endogenous inhibitors, TIMP (tissue inhibitors of metalloproteinase), which interact with cytokines. For example, TGF-beta 1 increases the level of TIMP-1 and decreases levels of MMP-1 and MMP-3, which counteracts degradation of ECM [12]. A significantly decreased level of TIMP-1 and an increased level of mRNA for MMP were noted in myomas subjected to treatment using GnRH-a, as compared to untreated tumours [13]. Moreover, Palmer et al. [14] observed augmented expression of MM11 , which degrades fibronectin in myomas, as compared to normal myometrium. MMP-2 and MMP- 9 behaves in a similar manner [15, 16], although Dou et al. [17] observed lower expression of MMP-1, $-2,-3$ and -9 in normal myometrium as compared to myomas. The highest expression developed in the course of luteal phase, dominated by progesterone even of earlier studies denied this information both in vivo and in vitro [4]. It was also noted that TGF-beta increased ability of chicken fibroblasts to incorporate fi- 
bronectin to ECM as well as augmented expression of receptor for the protein as well as for collagen [18].

Upon application of progesterone in human stromal cells of myoma increase production of TGF-beta, which in turn induces repression of MMP-3 and MMP-7 production [19]. The two enzymes degrade fibronectins. TGF-beta elevates also expression of collagen and enhances its incorporation to ECM [20].

Within uterus, morphological alterations are linked to ECM which should clearly distinguish from alterations which accompany myoma. They include (21):

1- Proliferation of stroma (stromatosis), or proliferation of multipotential mesenchymal cells with potential for transformation to various morphological forms under effect of hormones. For example, in the oestrogendependent phase small, elongated cells are observed with oval cell nucleus, rich in chromatin. Their excessive proliferation accompanied by no traits of atypia is defined as stromatosis s. hyperplasia stromalis endometrii. A myometrium-compressing tumour may be formed or a tumour protruding to uterine cavity (stromatosis nodularis), i.e. an endometrial stromal nodule. It is a benign tumour and it contains few mitoses and an insignificant atypia;

2- Endometrial stromal sarcoma (low grade endometrial stromal sarcoma) demonstrating a positive reaction for presence of ER and PG receptors and for CD 10. Its mitotic activity is low but the tumour infiltrates myometrium, penetrates lymphatic vessels (which is reflected in its older name of myosis stromalis endolymphatica) and tissue fissures. Around 3 mitoses can be noted per high power microscope field (HPMF);

3- Undifferentiated endometrial sartoma of highly malignant character (high grade undifferentiated endometrial sarcoma), manifesting over 10 mitoses per HPMF;

4- Tumours of an equivocal malignant character or smooth muscle tumours of unknown or undetermined malignant potential (SMTUMP);

5- A mesodermal mixed tumour (tumor mixtus mesodermalis malignus $s$. carcinosarcoma) originating from epithelial primordia of Muller ducts and of connective tissue and, thus, containing elements of planocellular adenocarcinoma or anaplastic carcinoma as well as traits of malignant mesenchymal tumours, such as, e.g., rhabdomyo - osteo - chondrosarcoma. Its sarcomatous portion manifests expression of, i.a., keratin [21].

\section{MYOMA AND LEIOMYOSARCOMA OF UTERUS}

Uterine leiomyoma (leiomyoma s. fibroid uteri) consists of smooth myocytes and contains reticular fibres, occasionally also collagen and elastic fibres, manifesting certain traits of fibroblasts due to their common mesenchymatic origin. Therefore, one can define it as a fibroid (fibroleiomyoma), which is difficult to distinguish from fibroma. It originates from smooth myocytes of the organ or from undifferentiated perivascular cells. It contains no capsule of connective tissue but it remains well demarcated $[1,21]$. It may undergo coagulative necrosis, calcification and hyaline degeneration. Coagulative necrosis remains well demarcated from the normal tissue, it contains shadows of cells and cell nuclei and its presence speaks in favour of myosarcoma manifestation even if the remaining microscope traits are poorly expressed [21]. In absence of necrosis the number of mitoses is critical for the diagnosis and tumours containing $<10$ mitoses /10 HPMF with no cell polymorphism carry good prognosis while tumours containing $>10$ mitoses/10 HPMF with no polymorphism represent malignant tumours. Moreover, tumours of a high polymorphism, with necrotic foci and low number of mitoses, e.g. 5/10 HPMF are regarded to be malignant.

Even if it is a non-malignant tumour, myoma occasionally behaves in a paradoxical way, e.g. it contains numerous mitoses (leiomyoma cellularis) and it may form implantation metastases to peritoneal cavity but only in the course of pregnancy. A morphological pattern of the metastasis mimics uterine myoma, resembles myosarcoma or SMTUMP [1]. Other investigators express opinion that the foci are not metastases but foci of diffuse metaplasia originating from uterus [4]. Penetration of neoplastic cells was observed also to veins of small pelvis, lymph nodes and to lungs, on the average 15 years after resection of the myoma from uterus [22].

In turn, leiomyosarcoma develops as a rule directly from myocytes and very seldom on the background of already existing myoma (as a secondary tumour). The cells manifest irregular arrangement, high atypia, frequently with presence of multinuclear cells and abnormal mitotic figures.

The cited data related to pathomorphosis of uterine myomas and leiomyosarcomas definitely require a comment. The allegation that uterine myomas develop in peritoneal cavity by multifocal metaplasia plainly contradicts the definition of metaplasia. Metaplasia represents transformation of a differentiated and mature tissue into another, also differentiated and mature tissue as a consequence of altered direction of differentiation of stem cells. Thus, it has nothing to do with neoplastic transformation. In a similar way the observation that uterine myoma, being a benign tumour, yields metastases to various organs and in a quite a distant time (in lungs on the average after 15 years) seems improbable. It should rather be accepted that the myomas developed from smooth muscle of bronchial tree or from muscularis of blood vessels or, which is highly probable, they represent hormonally stimulated proliferation of tumour cells but in a place distinct than uterus. Thus, it would represent an autocrine self-stimulation. In addition to the ovarian hormone, uterine myomas are known to to produce oestrogen, manifest expression of oestrogen synthase, aromatase and transform the circulating androgen to oestrogen in situ, which is sufficient for the growth of tumour, independently oestrogen, with involvement of aromatase, coded by CYP 19 gene, localised on chromosome 15, locus q21.1 and including 10 exons [18]. Genes of steroidogenesis enzymes play important role among factors which control cell cycle. It is also highly probable to represent the effect of neoplastic selforganisation, based on thermodynamic disturbances (II principle of thermodynamics). 


\section{DISSIPATIVE NATURE OF TUMOURS}

In line with principles of thermodynamics, cells which are in a dissipative (energy spreading) condition may return to equilibrium, die or begin living manner more effective from the point of view of energy dissipation [23]. In such cases we talk about neoplastic transformation. Thus, the only sufficient cause of tumour development involves the inner dissipative condition of cells, to which can lead multiple, variable but only indispensable factors. In physics the dissipative condition carries the name of bifurcation point, positioned within the range of unstable conditions distant from the inner balance of the system. Thus, a tumour represents a dissipative structure and, as follows from the IInd principle of thermodynamics, it is a self-organizing new biological structure. This requires higher energy investment or the so called negative entropy and, thus, the entire energy expenditure is used in the cells for its proliferation, the remains being insufficient for cell differentiation. This is particularly true of malignant tumours.

According to Klimek, a dissipatogenic space exists around the neoplastic tumour in which genetic changes develop which do not permit the cell yet to acquire a neoplastic phenotype but which are sufficiently detectable to take them into account in therapeutic attempts [24]. Development of the neoplastic phenotype represents a very complex phenomenon, linked, i.e. to cell differentiation, apoptosis, growth, angiogenesis, migration, adhesion, cell cycle control and, finally, cell death. For example, if a single mutated gene in the form of DNA will be included into apoptotic bodies, formed due to apoptosis of a neoplastic cell, and it will be incorporated to a normal cell it may continue to replicate, explaining formation of tumour metastases [24]. Moreover, progression of a tumour and development of metastases was noted to be linked to expression of metallothioneins (MT) as an anti-apoptotic factor. Intensity of MT expression in repair of damaged DNA allows to conclude on intensity of genetic lesions developing in the neoplastic cell and in cells surrounding them and for the potential for interference with the use of apoptotic bodies originating from neoplastic cells in contact with cells of healthy tissue margins. Accordingly, expression of MT increases not only in cells of endometrial cancer but also in the healthy epithelium which surrounds the tumour and in uterine smooth muscles in a direct vicinity of the infiltration [24].

\section{MYOMA AND LEIOMYOMA - PRESENCE OF DIS- ORIC ZONE}

A group of tumours also exists, including myomas and myosarcomas, with reciprocally overlapping traits which are termed the border-line tumours and which present a significant challenge for histopathologists $[4,18]$. In addition it has not been proven whether one of the forms, i.e. myomas provide a starting point for development of the other, i.e. myosarcomas although it should be stressed that myosarcomas produce myofibrils and, thus, they behave like myoblasts. In the cases in which myocytes differentiate to neoplastic myocytes the matter is simpler than in cases when the cells differentiate toward cells of endometrial stroma. The former are accompanied by blood vessels of thick, branching walls the latter are supplied mainly by thin-wall capillaries of the arborescent pattern. Finally, the differentiation can be facili- tated by immunocytochemical staining for desmin, cytokeratin, CD 10 and caldesmon [22]. In stromal tumours which infiltrate myometrium prognosis is unfavourable but in a typical myoma it is good, even in presence of infiltrations. Among other traits, potentially malignant character of a tumour may be indicated in a directly proportional manner by presence of coagulative necrosis in the tumour, focal or widespread cell atypia (pleomorphism, nuclear hyperchromasia) and, as earlier mentioned, the mitotic index. On the other hand, appearance of haemorrhagic foci, e.g. in the course of pregnancy or hormonal therapy, does not point to tumour malignancy and shows no relationship to tumour necrosis. It should also be stressed that high mitotic index in myomas rarely only correlates with its malignant character. Similar criteria are applied in the seldom seen form of the tumour, i.e. leiomyomyxoma.

The important diagnostic variable involves expression of $\mathrm{Ki}-67$, noted in $50 \%$ females with myosarcomas, in $8 \%$ females with myomas and absent in SMTUMP type tumours $[4,22]$. In a similar manner expression of cdks (cdk2 and edk2), cyclins $\mathrm{E}$ and $\mathrm{A}, \mathrm{p} 16$ protein, metallothionein 2 and PG receptor were higher in myosarcoma than in SMTUMP [18]. In both types of tumours AgNORs [25], PCNA, HER2/neu, p53 protein and MDM -2 were studied and the indiced proved to be of a high diagnostic value. In serum levels of lactic dehydrogenase (LDH) and of isoenzymes, particularly of LDH3 type, were higher in myosarcoma than in myomas. Moreover, myosarcoma does not change its size following therapy with analogues of gonadotropin releasing hormone, in contrast to myomas [26]. Protooncogene c-kit is expressed only in the former tumour which opens perspective for treatment of myosarcomas using tyrosine kinase inhibitors. Zhai et al. [27] also noted in myosarcoma loss of heterozygosity in nine loci (p53, RBI, DCC, NNM23, WTI, D14S67, p16, DPC4, PTCH) within or close to tumour suppressor genes in 20 patients. The conclusion follows that not an individual observation but only multidirectional studies, particularly immunocytochemical ones, provide the most accurate diagnostic and predictive criteria.

According to Domagała [21] neoplasmas are not static tumours and undergo changes in time depending on the degree of genetic instability, influences of environment and therapy. Frequently a tumour originates from one clone of cells, which subsequently differentiate in two directions, e.g. in the epithelial and mesenchymatic directions as it happens in carcinosarcoma. Therefore, it is not tumour histogenesis or cellular origin but direction of differentiation of the cells which is more important. The latter may be evaluated using a microscope, immunocytochemical and molecular studies. Tumour cells may exhibit uniform or variable expression of phenotypic traits and, therefore, leiomyosarcoma represents a malignant tumour which not only originates from smooth muscle cells but differentiates toward smooth muscles while carcinosacroma involves a tumour which differentiates in the epithelial and mesenchymatic direction although it need not to originate from the cells. Therefore, the direction of differentiation is more important for a clinician than the hypothetical origin of the tumour [21].

Behaviour of myomas and particularly of those which undergo coagulative necrosis may be ompared to the pattern of the so called knot-type inflammatory focus, composed of 5 zones, including the central necrosis surrounded by infiltrate 
of granulocytes with multilobar cell nuclei, zone of hyperaemia, multifocal inflammatory belt (histiocytes, lymphocytes, plasmacytes) and, first of all, the marginal band or the disoric zone (tissue oedema and secondary injury to healthy tissues). For oncology the most significant is the marginal band beyond which the tissue should be healthy. In analogy to the inflammatory process, the zone should be termed the disoric neoplastic zone, which no longer contains neoplastic cells but its cells, nevertheless, exhibit several tumourigenic traits, e.g. increased activity of telomerase, IL-8, vascular endothelium growth factor and lowered levels of EGF $[4,18]$. Following a variable period of rest the cells begin the stages of initiation, promotion and progression and may provide a site of relapse of the tumour or of metastases. For example, augmented activity of telomerase inhibits apoptosis and in this way promotes proliferation of tumour cells [28]. The increased activity of telomerase in the disoric zone was observed by Madej et al. [29]. This might explain why myosarcomas of less than $5 \mathrm{~cm}$ in diameter infrequently are aggressive and those of up to $3 \mathrm{~cm}$ in diameter form no metastases [24].

\section{SUMMARY}

ECM-extracellular matrix and not tumour parenchyma as a criterion of categorization, the tumours can be divided into two groups, i.e. those which produce stroma (non-epithelial malignant tumours) or sarcomas and the tumours which take advantage of the local tissue stroma (malignant epithelial tumours) or carcinomas. Involvement of stroma is noted also within the reciprocal relationship between stroma and tumour cells, which has been described in detail on the example of uterine myoma. ECM also "collaborates" with CAM (cell adhesion molecules), particularly in development of neoplastic metastases.

Pathomorphosis of myomas, myosarcomas and of "stromal" uterine tumours was described with particular attention given to differential diagnosis and the resulting for the clinic predictive and prognostic implications. A probable mechanism of neoplasia based on dissipative structures of cells was presented and introduction of a disoric zone of a tumour, i.e. of a marginal zone between the tumour and the morphologically normal tissue was suggested. The zone seems extremely important in prediction and prognosis related to relapse and/or tumour metastases.

\section{REFERENCES}

[1] Kruś S, Skrzypek-Fakhoury E. Clinical pathomorphology. PZWL Press, Warszawa, 1996.

[2] Madej JA. The part of stroma connective tisuue in formation pathomorphlogical picture of neoplasmatic growth. Med Vet 2000; 56: $18-22$

[3] Kawiak J, Zabel M. Seminars from cytophysiology. Urban \& Partner, Wrocław, 2002.

[4] Schwartz EP, Kelly MG. Malignant tranformation of myomas: myth or reality? Obstet Gynecol Clin N Am 2006; 33: 183-98.

[5] Wójcik C.The part of adhesive particles in chosen the hyperplastic processes. Post Biol Kom 1997; 24: 145-71.

[6] Kawaguchi K, Fuji S, Konishi I, et al. Mitotic activity in uterine leiomyomas during the menstrual cycle. Am J Obstet Gynecol 1989; 160: 637-18.

[7] Steward EA, Friedman AJ, Peck K, et al. Relative overexpression of collagen type 1 and collagen type 3 messenger ribonucleic acids by uterine leiomyomas during the propliferative phase of the menstrual cycle. J Clin Endocrinol Metab 1994; 79: 900-6.

[8] Arici A, Sozen I. Transforming growth factor-beta3 is expressed at high levels in leiomyoma where it stimulates fibronectin expression and cell proliferation. Fertil Steril 2000; 73: 1006-11.

[9] Fujita M. Histological and biochemical studies on collagen in human uterine leiomyomas. Hokkaido Igaku Zasshi 1985; 60: 602-15.

[10] Puistola U, Ristili L, Ristili J, et al. Collagen metabolism in gynecologic patients: changes in the concentration of the aminoterminal propeptide of type 3 procollagen in serum. Am J Obstet Gynecol 1990; 163: 1276-81.

[11] Wolanska M, Sobolewski K, Drozdzewicz M, et al. Extracellular matrix components in uterine leiomyoma and their alteration during the tumor growth. Mol Cell Endocrinol 1998; 189: 145-52.

[12] Ma C, Chegini N. Regulation of matrix proteinases (MMPs) and their tissue inhibitors in human myometrial smooth muscle cells by TGF beta-1. Mol Hum Reprod 1999; 5: 950-4.

[13] Milman D, Zalel Y, Biran H, et al. Unsuspected uterine leiomyosarcoma discovered during treatment with a gonadotropin-releasing hormone analogue: a case report and literature review. Eur J Obstet Gynecol Reprod Biol 1998: 76: 237-40.

[14] Palmer SS, Haynes-Johnson D, Diehl T, et al. Increased expression of stromelysin $3 \mathrm{mRNA}$ in leiomyomas (uterine fibroids) compared with myometrium. J Soc Gynecol Invest 1998; 5: 203-9.

[15] Hulboy DL, Rudoph LA, Matrisian LM. Matrix metalloproteinases as mediators of reproductive function. Mol Hum Reprod 1997; 3: $27-45$.

[16] Ignaki N, Ung L, Otani T, et al. Uterine cavity matrix metalloproteinases and cytokines in patients with leiomyoma, adenomyosis and endometrial polyp. Eur J Obstet Gynecol Reprod Biol 2003; 111: 197-203.

[17] Dou Q, Tarnuzzer WR, Wiliams RS, et al. Differential expression of matrix proteinases and their tissue inhibitors in leiomyomata: a mechanism for gonadotropin releasing hormone agonist - induced tumor regression. Mol Hum Reprod 1997; 3: 1005-14

[18] Sozen I, Arici A. Cellular biology of myomas: ineraction of sex steroids with cytokines and growth factors. Obstet Gynecol Clin N Am 2006; 33: 41-58.

[19] Bruner KL, Rodgres WH,Gold LI, et al. Transforming growth factor beta mediates the progesterone suppression of an epithelial metalloproteinase by adjacent stroma in the human endometrium. Proc Natl Acad Sci USA 1995; 92: 7362-6.

[20] Ignotz RA, Massague J. Transforming growth factor beta stimulates the expression of fibronectin and collagen and their incorporation into the extracellular matrix. J Biol Chem 1986; 261: 4337-45.

[21] Stachura J, Domagała W. Patologia-znaczy słowo o chorobie. Pathology - means word about disease. Vol. I and II, PAU, Kraków, 2003.

[22] Kayser K, Zink S, Schneider T, et al. Benign metastasizing leiomyomas of the uterus: documentation of clinical, immunohistochemical and lectin-histochemical data of ten cases. Virchows Arch 2000; 437: 284-92.

[23] Klimek R, Madej JM, Sieroń A. Cancer and disease neoplasmic tumours. R. Klimek Press, Kraków, 2006.

[24] Klimek M, Wicherek Ł, Gałązka K, et al. Cycle dependent expression of endometrial metallothionein. Neuroendocrinol. Latters 2005; 26: 663-66.

[25] Madej P, Madej JA, Plewka A, et al. Evaluation of nucleolar organizer region (NOR) parameters in the uterine leiomyoma. Path Res Pract 2005; 201: 587-92.

[26] Meyer WR, Mayer AR, Diamond MP, et al. Unsuspected leiomyosarcoma: treatment with a gonadotropin-releasing hormone analogue. Obstet Gynecol 1990; 75: 529-32.

[27] Zhai YL, Nikaido T, Orii A, et al. Frequent occurence of loss of heterozygosity among tumour supressor genes in uterine leiomyosacroma. Gynecol Oncol 1999: 75: 453-549.

[28] Hsu YL, Kuo PL, Lin CC. The proliferative inhibition and apoptotic mechanism of Saikosgonium D in human non-small cell lung cancer A549 cells. Life Sci 2004; 75: 1231-42.

[29] Madej P, Plewka A, Madej JA, et al. Immunohistochemical localization of telomerase in myomas and in uterine myometrium -in press. 